\title{
Heat and Mass Transfer on MHD Viscoelastic Fluid Flow in the Presence of Thermal Diffusion and Chemical Reaction
}

\author{
Amos O. Popoola ${ }^{1 *}$, Ismail G. Baoku ${ }^{2}$ and Bakai I. Olajuwon ${ }^{3}$ \\ ${ }^{*}$ Department of Mathematical and Physical Sciences, Osun State University, \\ P.M.B.4494, Osogbo, Nigeria \\ ${ }^{2}$ Department of Physical Sciences, Bells University of Technology, \\ P.M.B. 1015, Ota, Nigeria \\ ${ }^{3}$ Department of Mathematics, Federal University of Agriculture, \\ P.M.B. 2240, Abeokuta, Nigeria
}

Email: bobdee11@yahoo.com

\begin{abstract}
The paper carries out an analysis on the momentum, heat and mass transfer characteristics in an incompressible magnetohydrodynamic non-Newtonian boundary flow of a viscoelastic fluid over a stretching sheet in the presence of thermal diffusion and chemical reaction. The partial differential equations governing the flow as well as heat and mass transfer features are converted into highly non-linear coupled ordinary differential equations by similarity transformations. The resulting differential equations are solved by using a shooting technique with fifth-order Runge-Kutta-Fehlberg integration scheme. The influence of magnetic interaction, variable thermal conductivity, viscoelastic, variable fluid viscosity, heat source/sink rate of chemical reaction, themal radiation and thermal diffusion parameters as well as Prandtl and Schmidt numbers are analyzed for velocity, temperature and concentration profiles. The wall shear stress, wall temperature and concentration gradients are also investigated for the problem. The fluid viscosity varies as an inverse function of velocity while the thermal conductivity assumes a linear and an inverse function of temperature and concentration respectively. The study shows that the thermal diffusion and thermal radiation parameters have opposite effects on the skin friction coefficient and wall concentration gradient. However, the rate of chemical reaction has similar influence on the skin friction coefficient and wall temperature gradient.
\end{abstract}

Keywords: Thermal diffusion, Thermal radiation, Chemical reaction, MHD, Viscoelastic fluid, Variable viscosity.

\section{INTRODUCTION}

The physics of simultaneous heat and mass transfer has attracted various researchers because of its various ranges of applications in science and technology such as food processing, polymer solutions, wet-bulb thermometer and in various fluid flows related engineering problems [1-7]. As a result of this prime importance of heat and mass transfer involving chemical reaction in non-Newtonian fluids, the study has received considerable attention in recent years. Viscoelastic flows are prevalent in numerous processes employed by the chemical engineering systems. Such flow exhibits both viscous and elastic properties, normal stresses and relaxation effects. A wide range of mathematical models have been developed to simulate the diverse hydrodynamic and magnetohydrodynamic behaviours of these nonNewtonian viscoelastic fluids [8-18].

The boundary layer flows of non-Newtonian fluids over a stretching sheet with heat and mass transfer are important in several areas such as paper production, glass fibber, extrusion process, wire drawing, crystal growing, hot rolling, food processing, electronic chips, movement of biological fluids, plastic manufacture and application of paints (Hayat and Qasim [19]). Bhargava et al. [20] studied the heat and mass transfer of boundary layer flow over a non-linear stretching sheet under the effects of different physical parameters. Analysis was carried out by Singh [21] to study heat source and radiation effects on an electrically conducting, twodimensional steady flow of an incompressible viscoelastic fluid past a stretching sheet in the presence of transverse uniform magnetic field. The hydromagnetic flow and heat transfer adjacent to a stretching vertical sheet with prescribed surface heat flux was investigated by Aman and Ishak [22]. Abbas and Hayat [23] considered the stagnation point with slip effects in heat transfer analysis for the boundary layer flow over a non-linear stretching sheet. The heat and mass transfer over a stretching sheet was further studied by several researchers including Pal and Mondal [24] who considered the effects of buoyancy and solutal buoyancy parameters. 
Also, the interaction between the conducting fluid and the applied magnetic field basically modifies the flow with attendant effects on such flow properties. The detailed nature of this interaction strongly depends on the orientation of the magnetic field. Understanding the dynamics of such conducting fluids has relevant applications in the advent of technology that involves the MHD devices, MHD power generators, possibility of thermonuclear power, nuclear engineering, e.t.c. Malashestty and Umavathi [25] examined two-phase MHD flow and heat transfer in the presence of buoyancy effects in an inclined channel for the situation where only one of the phases is electrically conducting. Malashetty et al. [26] further investigated the magnetoconvection of two immiscible fluids in a vertical enclosure. Cortell [27] studied the flow and heat transfer of an electrically conducting second grade fluid subject to suction and a transverse magnetic field over a stretching sheet. Similarly, the heat and mass transfer in a viscoelastic fluid flow with magnetic field and heat source or sink through a porous medium on a stretching sheet was examined by Seddeek [28]. Emad and Mohamed [29] considered the effect of blowing/suction on hydromagnetic heat transfer by mixed convection with internal heat generation/absorption.

The study of MHD with mass and heat transfer in the presence of thermal radiation and diffusion has attracted the attention of a large number of scholars due to its diverse applications. Grosan and Pop [30] examined the effect of thermal radiation in a vertical channel on fully developed mixed convection flow. Srinivas and Muthuraj [31] used the homotopy analysis method to investigate thermal radiation and space porosity effects on MHD mixed convection flow in a vertical channel. The combined influence of chemical reaction, radiation and MHD on mixed convection heat and mass transfer along a moving surface was considered by Joshi and Kumar [32]. Thermal diffusion or Soret effect corresponds to species differentiation developing in an initial homogeneous mixture submitted to a thermal gradient. Thermal diffusion arises when the mass flux contains a term that depends on the temperature gradient. Eldahe et al. [33] analyzed the thermal diffusion and diffusion-thermo effects on mixed free-convection and mass transfer boundary layer for non-Newtonian fluid with temperature-dependent viscosity. Anghel et al. [34] examined the effects of Dufour and Soret on free convection boundary layer over a vertical surface embedded in a porous medium. The combined effects of Soret and Dufour diffusion and porous impedance on laminar MHD mixed convection heat and mass transfer on an electrically conducting Boussinesq fluid from a vertical stretching surface in a Darcian porous medium under uniform transverse magnetic field was studied by Beg et al. [35].

For most of the above investigations, the physical properties of the ambient fluid were assumed to be constant. However, it is obvious in [36-40] that these physical properties of the ambient fluid may change with temperature and so, the fluid viscosity may no longer be assumed constant. Pantokratoras [41] has presented some results on the variable viscosity of flow and heat transfer to a continuous moving flat plate. Mukhopadhayay and Layek [42] examined the thermal radiation and variable fluid viscosity effects on free convective and heat transfer past a porous stretching surface. Prasad et al. further carried out an analysis on the effect of variable viscosity on MHD viscoelastic fluid flow and heat transfer characteristics over a stretching sheet. Siddappa and Abel [43] also considered the variable fluid properties which depend on high temperature past a stretching sheet.

Motivated by all these studies, the present work intends to investigate the heat and mass transfer on Magnetohydrodynamics and viscoelastic fluid flow over an impermeable long continuous stretching sheet with variable viscosity and thermal conductivity in the presence of thermal diffusion and chemical reaction.

\section{MATHEMATICAL MODEL}

\subsection{Governing equations}

Consider a steady two-dimensional flow of an incompressible magnetohydrodynamic second grade viscoelastic fluid past an impermeable long continuous sheet with heat generation/absorption and thermal diffusion in the presence of thermal radiation and chemical reaction. The sheet which issues from a slot coincides with the plane $y=0$ and the flow being confined to $y>0$. The moving of the continuous sheet is taken up by a wind-up roll. It is assumed that after the initiation of motion, a certain time has elapsed so that the steady conditions hold. Taking the $x$-axis in the direction of the main flow along the sheet and $y$-axis normal to it, the origin is located at the slit through which the sheet is drawn through the fluid medium. Two equal and opposite forces are applied along the $x$-axis so that the sheet is stretched, while keeping the origin fixed. A magnetic field of strength $B_{0}$ is applied in the positive $y$-direction. The continuous sheet varies with a velocity according to a linear form $u=u_{w}=b x$ and it is subjected to a prescribed surface temperature, i.e. at the solid surface. The fluid moves in the $x$-direction with a velocity ( $u$-component) equals to the velocity of the fluid surface, whereas at increasing distance from the surface, the velocity of the fluid in the $x$-direction approaches to zero asymptotically.

Assumptions:

a. Any flow disturbance created by the roll is neglected;

b. An observer fixed in space will notice that the boundary layer on the sheet originates at the slot and grows in the direction of the motion of the sheet;

c. It is assumed that the flow approaches the sheet with zero angle of incidence and the sheet issues from a slot at the origin;

$\mathrm{d}$. The fluid viscosity is assumed to vary as a function of temperature;

e. The magnetic Reynolds number is assumed to be small so that the induced magnetic field is neglected in comparison to the applied magnetic field;

f. There is negligible applied electric field so that Hall effect and Joule heating are neglected.

The fluid is thermodynamically compatible and the constitutive equation is given by Rivlin and Erickson [44]. Invoking the usual boundary layer approximation under the aforementioned assumptions, the governing equation for momentum, heat and mass transfer in Walters' liquid B model in the presence of variable fluid viscosity and thermal conductivity can be taken as considered by [45]:

$\frac{\partial u}{\partial x}+\frac{\partial v}{\partial y}=0$ 
$u \frac{\partial u}{\partial x}+v \frac{\partial u}{\partial y}=\frac{1}{\rho_{\infty}} \frac{\partial}{\partial y}\left(\mu \frac{\partial u}{\partial y}\right)-\frac{\sigma B_{0}^{2}}{\rho_{\infty}} u$

$-k_{0}\left\{v \frac{\partial^{3} u}{\partial y^{3}}+u \frac{\partial^{3} u}{\partial x \partial y^{2}}+\frac{\partial u}{\partial x} \frac{\partial^{2} u}{\partial y^{2}}-\frac{\partial u}{\partial y} \frac{\partial^{2} u}{\partial x \partial y}\right\}$

$\rho_{\infty} C_{p}\left(u \frac{\partial T}{\partial x}+v \frac{\partial T}{\partial y}\right)=\frac{\partial}{\partial y}\left(K(T) \frac{\partial T}{\partial y}\right)+Q_{s}\left(T-T_{\infty}\right)-\frac{\partial q_{r}}{\partial y}$

$u \frac{\partial C}{\partial x}+v \frac{\partial C}{\partial y}=D_{m} \frac{\partial^{2} C}{\partial y^{2}}+\frac{D_{m} K_{T}}{T_{m}} \frac{\partial^{2} T}{\partial y^{2}}-r\left(C-C_{\infty}\right) *$

where $u$ and $v$ are the fluid velocity components in the streamwise $x$ and cross-stream $y$-directions respectively; $k_{0}$ is the coefficient of viscoelasticity, $\sigma$ is the electrical conductivity, $\beta_{0}$ is the magnetic field strength, $C_{p}$ is specific heat at constant pressure. Here, $\rho_{\infty}$ is the constant fluid density and $\mu$ is the coefficient of viscosity which is considered to vary as an inverse function of temperature according to Lai and Kulacki [37] as follows:

$\frac{1}{\mu}=\frac{1}{\mu_{\infty}}\left[1+\gamma\left(T-T_{r}\right)\right] \quad$ i.e. $\frac{1}{\mu}=a\left(T-T_{\infty}\right)$

where $a=\frac{\gamma}{\mu_{\infty}}, T_{r}=T_{\infty}+\frac{1}{\gamma}$ and $a>0$ corresponds to liquids and $a<0$ is for gases.

$T$ is the ambient temperature, $T_{\infty}$ and $\mu_{\infty}$ are the constant values of the temperature and dynamic viscosity far away from the sheet respectively.

This type of fluid variable viscosity has been used by [39, 46] and is more appropriate for the present investigation as it is valid for wide temperature range.

The temperature-dependent thermal conductivity $K(T)$ relationship considered in this study takes the form [47]:

$K(T)=K_{\infty}\left(1+\frac{\varepsilon}{\Delta T}\left(T-T_{\infty}\right)\right)$

where $\Delta T=T_{w}-T_{\infty}, T_{w}$ is the sheet temperature, $\varepsilon$ is the small parameter and $K_{\infty}$ is the conductivity of the fluid far away from the sheet.

Using Rosseland approximation, the third term $q_{r}$ on the right hand side of equation (3) represents the radiative heat flux as given by [48]:

$q_{r}=\frac{-4 \sigma^{*}}{3 K^{*}} \frac{\partial T^{4}}{\partial y}$

where $\sigma^{*}$ and $K^{*}$ are the Stefan-Boltzmann constant and the mean absorption coefficient respectively.

It is assumed that the differences within the flow are such that the term $T^{4}$ may be expressed as a linear function of temperature by expanding $T^{4}$ in a Taylor series about $T_{\infty}$ as:

$T^{4}=T_{\infty}^{4}+4 T_{\infty}^{3}\left(T-T_{\infty}\right)-6 T_{\infty}^{2}\left(T-T_{\infty}\right)^{2}+\cdots$ and neglecting the higher order terms beyond the first degree in $\left(T-T_{\infty}\right)$ to obtain $T^{4} \cong 4 T_{\infty}^{3}-3 T_{\infty}^{4}$. Substituting this expression of $T^{4}$ in equation (7) to get:

$q_{r}=\frac{-16}{3} \frac{\sigma^{*}}{K^{*}} T_{\infty}^{3} \frac{\partial T}{\partial y}$

The term involving $Q_{s}$ in equation (3) represents the temperature-dependent volumetric rate of heat source when $Q_{s}>0$ and heat sink when $Q_{s}<0$ which correspond to the situation of exothermic and endothermic reactions respectively. In equation (4), the parameter $r_{0}$ represents the rate of chemical reaction, $K_{T}$ stands for the thermal diffusion parameter, $T_{m}$ is the mean fluid temperature and $D_{m}$ is the coefficient of mass diffusivity.

Substituting the equations (5), (6) and (9) in equations (2), (3) and (4), we have:

$$
\begin{aligned}
& u \frac{\partial u}{\partial x}+v \frac{\partial u}{\partial y}=\frac{1}{\rho_{\infty}} \frac{\partial}{\partial y}\left(\frac{\mu_{\infty}}{1+\gamma\left(T-T_{\infty}\right)} \frac{\partial u}{\partial y}\right)-\frac{\sigma B_{0}^{2}}{\rho_{\infty}} u \\
& -k_{0}\left\{v \frac{\partial^{3} u}{\partial y^{3}}+u \frac{\partial^{3} u}{\partial x \partial y^{2}}+\frac{\partial u}{\partial x} \frac{\partial^{2} u}{\partial y^{2}}-\frac{\partial u}{\partial y} \frac{\partial^{2} u}{\partial x \partial y}\right\} \\
& \rho_{\infty} C_{p} u \frac{\partial T}{\partial x}+\left(\rho_{\infty} C_{p} v-\frac{k_{\infty} \varepsilon}{\Delta T} \frac{\partial T}{\partial y}\right) \frac{\partial T}{\partial y}=k_{\infty}\left(1+\frac{\varepsilon}{\Delta T}\left(T-T_{\infty}\right)\right) \frac{\partial^{2} T}{\partial y^{2}} \\
& +Q_{s}\left(T-T_{\infty}\right)-\frac{16 \sigma^{*} T_{\infty}^{3}}{3 K^{*}} \frac{\partial^{2} T}{\partial y^{2}}
\end{aligned}
$$

\subsection{Boundary conditions}

Considering the effect of stretching of the momentum, thermal and species concentration boundary surface causing the flow along $x$ - direction, the following appropriate boundary conditions on velocity, temperature and species concentration equations are employed:

$$
\begin{aligned}
& u=u_{w}=b x, v=0, T=T_{w}=T_{\infty}+A\left(\frac{x}{l}\right), \\
& C=C_{w}=C_{\infty}+B\left(\frac{x}{l}\right) \text { at } y=0 \\
& u \rightarrow 0, u_{y} \rightarrow 0, T \rightarrow T_{\infty}, C \rightarrow C_{\infty} \quad \text { as } y \rightarrow \infty
\end{aligned}
$$

where $b$ is a constant known as stretching rate, $A$ and $B$ are constants, $l$ is the characteristic length.

It is very important to note that equation (10) is a thirdorder partial differential equation in $u$ whereas the number of prescribed conditions on $u$ is given by equation (12) are two. Augumenting the boundary conditions according to Rajagopal [49, 50] and Mahapatra and Gupta [41] to have a unique solution of the equation, the order of the governing equation now equals to the number of prescribed boundary conditions. 


\subsection{Non-Dimensionalization analysis}

The following transformations are employed to examine the flow regime adjacent to the plate:

$$
u=b x f^{\prime}(\eta), v=-\left(b v_{\infty}\right)^{1 / 2} f(\eta), \eta=\sqrt{\frac{b}{v_{\infty}}} y
$$

$\left(T-T_{\infty}\right)=\left(T_{w}-T_{\infty}\right) \theta(\eta)$

where $\left(T_{w}-T_{\infty}\right)=A\left(\frac{x}{l}\right)$

$\left(C-C_{\infty}\right)=\left(C_{w}-C_{\infty}\right) \varphi(\eta)$ where $\left(C_{w}-C_{\infty}\right)=B\left(\frac{x}{l}\right)$

Clearly, this implies that $\Delta T=\left(T_{w}-T_{\infty}\right)=A\left(\frac{x}{l}\right)$, $\Delta C=\left(C_{w}-C_{\infty}\right)=B\left(\frac{x}{l}\right)$ and that equation (6) which expresses the temperature-dependent thermal conductivity turns out to be $x$-independent. Using a scaled $\eta$-dependent temperature and species concentration in the form of $\theta(\eta)=\frac{\left(T-T_{\infty}\right)}{\Delta T}$ and $\varphi(\eta)=\frac{\left(C-C_{\infty}\right)}{\Delta C}$ respectively, the condition that the $x$-variation of $T$ and $C$ in the fluid is the same as that along the sheet is imposed. Thus, employing these transformations, equation (1) is identically satisfied and equations $(2)-(4)$ reduce to:

$$
\begin{aligned}
& {\left[\theta(\eta)-\theta_{r}\right]^{2} f^{\prime 2}(\eta)-\left[\theta(\eta)-\theta_{r}\right]^{2} f(\eta) f^{\prime \prime}(\eta)} \\
& +\theta_{r}\left[\theta(\eta)-\theta_{r}\right]^{2} f^{\prime \prime \prime}(\eta)-\theta_{r} f^{\prime \prime}(\eta) \theta^{\prime}(\eta) \\
& +k_{1}\left[\theta(\eta)-\theta_{r}\right]^{2}\left[2 f^{\prime}(\eta) f^{\prime \prime \prime}(\eta)-f(\eta) f^{i v}(\eta)-f^{\prime \prime 2}(\eta)\right] \\
& +\operatorname{Mn}\left[\theta(\eta)-\theta_{r}\right]^{2} f^{\prime}(\eta)=0 \\
& {[1+\varepsilon \theta(\eta)] \theta^{\prime \prime}(\eta)+\varepsilon \theta^{\prime 2}(\eta)} \\
& +\operatorname{Pr} R_{0}\left[f(\eta) \theta^{\prime}(\eta)-\left(f^{\prime}(\eta)-\beta\right) \theta(\eta)\right]=0 \\
& \varphi^{\prime \prime}(\eta)+\frac{S c}{2} \eta f^{\prime}(\eta) \varphi^{\prime}(\eta)+S c f(\eta) \varphi^{\prime}(\eta) \\
& +\operatorname{Sr} \theta^{\prime \prime}(\eta)-r S c \varphi(\eta)=0
\end{aligned}
$$

The corresponding boundary conditions (12) are transformed into:

$$
\begin{aligned}
& f^{\prime}(\eta)=1, f(\eta)=0, \theta(\eta)=1, \varphi(\eta)=1 \quad \text { at } \quad \eta=0 \\
& f^{\prime}(\eta)=0, f^{\prime \prime}(\eta)=0, \theta(\eta)=0, \varphi(\eta)=0 \text { at } \eta \rightarrow \infty
\end{aligned}
$$

where $k_{1}=\frac{k_{0} b}{v_{\infty}}$ is the viscoelastic parameter, $\theta_{r}=\frac{1}{\gamma\left(T-T_{\infty}\right)}$ is the fluid viscosity parameter which is negative for liquids, $M n=\frac{\sigma B_{0}^{2}}{\rho_{\infty} b}$ is the magnetic parameter, $\operatorname{Pr}=\frac{\mu C_{p}}{k_{\infty}}$ is the
Prandtl number, $\beta=\frac{Q}{\rho_{\infty} C_{p} b} \quad$ is the heat source/sink parameter, $R_{0}=\frac{16 \sigma^{*} T_{\infty}^{3}}{3 k^{*} k_{\infty}}$ is the thermal radiation parameter, $S c=\frac{v_{\infty}}{D_{m}}$ is the Schmidtl number, $S r=\frac{k_{T}\left(T_{w}-T_{\infty}\right)}{b T_{m}\left(C_{w}-C_{\infty}\right)}$ is the thermal diffusion parameter and $r=\frac{r_{0}}{b}$

Investigations of flow behaviour, heat and mass transfer would be carried out by analyzing the dimensionless local shear stress $\tau_{w}$ (skin friction coefficient), rates of heat and mass transfer coefficients at the wall which are Nusselt $(\mathrm{Nu})$ and Sherwood $(S h)$ numbers respectively. These quantities are defined as follows:

$$
\begin{aligned}
& \tau=\frac{\tau^{*}}{\mu b x \sqrt{b / v_{\infty}}}=f^{\prime \prime}(\eta) \text { at } \eta=0 ; \\
& N u=\frac{-h}{\left(T_{w}-T_{\infty}\right)} T_{y}=-\theta(\eta) \text { at } \eta=0 ; \\
& S h=\frac{-h}{\left(C_{w}-C_{\infty}\right)} C_{y}=-\varphi(\eta) \text { at } \eta=0 ;
\end{aligned}
$$

\section{COMPUTATIONAL PROCEDURE}

Equations (14) - (16) constitute a highly non-linear coupled boundary value problem of fourth-order in $f$, second-orders in $\theta$ and $\varphi$. Since the exact solution does not seem to be feasible for complete set of equations (14) - (16), an effective numerical shooting technique with fifth-order Runge-KuttaFehlberg integration algorithm is therefore developed. The highly coupled boundary value problem in equations (14) (16) has been reduced to a system of eight simultaneous ordinary differential equations of first-order for eight unknowns following the method of superposition [51] by assuming $f=f_{1}, f^{\prime}=f_{2}, f^{\prime \prime}=f_{3}, f^{\prime \prime \prime}=f_{4}, \quad \theta=\theta_{1}$, $\theta^{\prime}=\theta_{2}, \varphi=\varphi_{1}, \varphi^{\prime}=\varphi_{2}$. To solve this system of equations, eight initial conditions are required while only two initial conditions $f(0), f^{\prime}(0)$ on $f$, one initial condition $\theta(0)$ on $\theta$ and one initial condition on $\varphi(0)$ on $\varphi$ are given. The third condition $f^{\prime \prime}(0)$ on $f$ has been deduced by applying initial condition (17) in equation (14). Yet, there are three initial conditions $f^{\prime \prime}(0), \theta^{\prime}(0)$ and $\varphi^{\prime}(0)$ which are not prescribed. However, the values of $f^{\prime}(\eta), \theta(\eta)$ and $\varphi(\eta)$ are known at $\eta=\infty$. Thus, the numerical shooting technique is applied whereby these three ending boundary conditions are employed to produce three known initial conditions at $\eta=0$. In order to select $\eta_{\infty}$, we begin with some initial guess values and solve the problem with some particular set of parameters to obtain $f^{\prime \prime}(0), \theta^{\prime}(0)$ and $\varphi^{\prime}(0)$. Therefore, we start with the initial approximation as $f_{3}(0)=a_{0}$, 
$\theta_{2}(0)=b_{0}$ and $\varphi_{2}(0)=c_{0}$. Let $a, b$ and $c$ be the correct values of $f_{3}(0), \theta_{2}(0)$ and $\varphi_{2}(0)$ respectively. The solution procedure is repeated with another larger value of $\eta_{\infty}$ until two successive values of $f^{\prime \prime}(0), \theta^{\prime}(0)$ and $\varphi^{\prime}(0)$ only differ after desired digit satisfying the limit of the boundary along $\eta$. The last value of $\eta_{\infty}$ is chosen as appropriate value for that particular set of parameters. Finally, the problem has been solved numerically utilizing fifth-order Runge-Kutta-Fehlberg integration scheme. It is important to note that convergence of the scheme largely depends on good guesses of the initial conditions in the shooting techniques.

\section{RESULTS AND DISCUSSION}

A numerical computation has been performed in order to analyze the influences of the various thermophysical parameters on the flow, heat and mass transfer of a viscoelastic fluid over a stretching sheet with variable fluid properties. The effects of various physical parameters on horizontal velocity, temperature and species concentration profiles for a linearly stretching sheet have been discussed and shown graphically in Figures (1) - (18). Figures (1 - 5), $(6-12)$ and $(13-20)$ show the velocity, temperature and species concentration fields respectively. Table 1 displays the local shear stress, rates of heat and mass transfer at the wall.

\subsection{Velocity profiles}

Figure 1 analyzes the effect of viscoelastic parameter $k_{1}$ on the horizontal velocity profile $f^{\prime}(\eta)$ with $\eta$. It is noticeable that the horizontal velocity profiles decrease with increasing value of viscoelastic parameter in the boundary layer. This confirms the fact that the influence of an increase in viscoelastic parameter is to reduce the horizontal velocity and this reduces the boundary layer thickness. Hence, this induces an increase in the absolute value of the velocity gradient at the surface. Figure 2 shows the influence of magnetic interaction parameter $M n$ on the horizontal velocity profile $f^{\prime}(\eta)$ with $\eta$. It is observed that an increase in the magnetic interaction parameter increases the horizontal velocity. This increases the boundary layer thickness and hence leads to a decrease in the absolute value of the velocity gradient at the surface. The effect of fluid viscosity parameter $\theta_{r}$ on horizontal velocity profiles $f^{\prime}(\eta)$ with $\eta$ for different values of viscoelastic physical parameters is depicted in Figure 3. It is observed that the horizontal velocity profiles $f^{\prime}(\eta)$ decreases with an increasing values of fluid viscosity parameter $\theta_{r}$. This is due to the fact that with an increasing value of fluid viscosity parameter $\theta_{r}$, the horizontal boundary layer thickness decreases which in turn results in the decrease in the horizontal velocity profile. Figure 4 and 5 are drawn to display the velocity profile $f^{\prime}(\eta)$ with $\eta$ for different values of variable thermal conductivity parameter and thermal radiation parameter $R_{0}$. It is observed that the effects of an increase in the value of thermal conductivity and thermal radiation parameters result in increase of the horizontal velocity profiles $f^{\prime}(\eta)$. This increases the horizontal boundary thickness. This is because of the fact that the viscoelastic fluid, with increasing variable thermal conductivity and high thermal radiation, is assumed to be less dense.

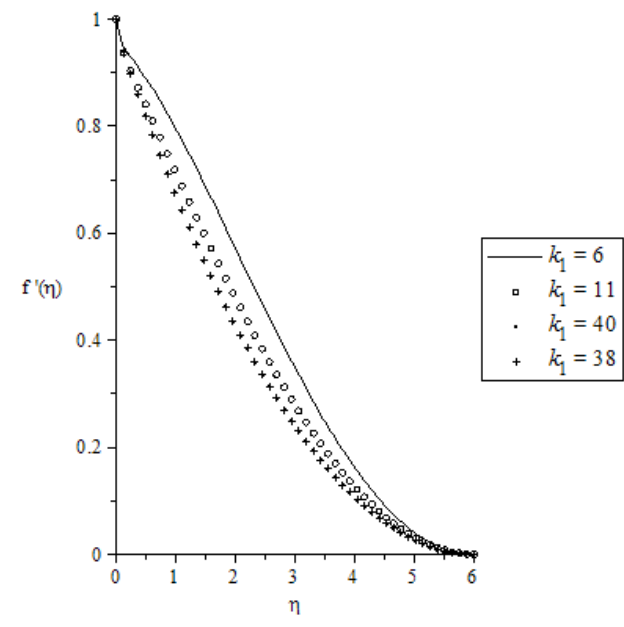

Figure 1. Velocity profiles for various values of $k_{1}$ when $M n=0.5, R_{0}=0.2, \theta_{r}=-1.0, \varepsilon=0.05$

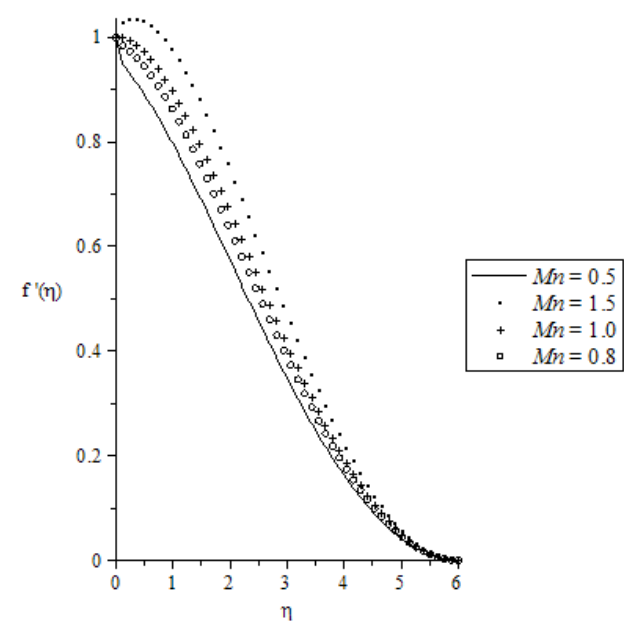

Figure 2. Velocity profiles for various $M n$ when $k_{1}=6.0$, $R_{0}=0.2, \theta_{r}=-1.0, \varepsilon=0.05$

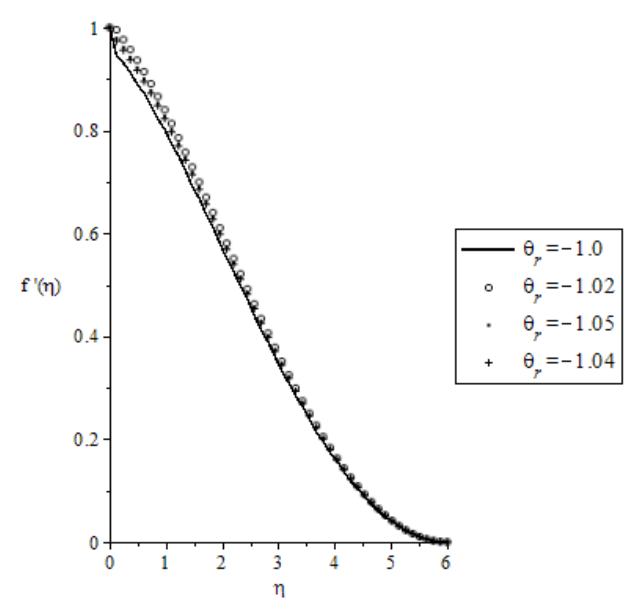

Figure 3. Velocity profiles for various values of $\theta_{r}$ when $k_{1}=6.0, R_{0}=0.2, M n=0.5, \varepsilon=0.05$ 


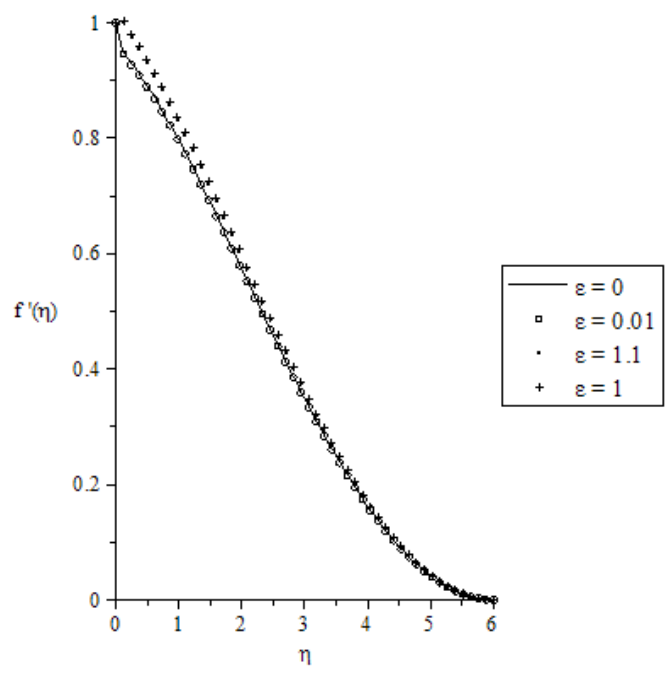

Figure 4. Velocity profiles for various values of $\varepsilon$ when $k_{1}=6.0, R_{0}=0.2, \theta_{r}=-1.0, M n=0.5$

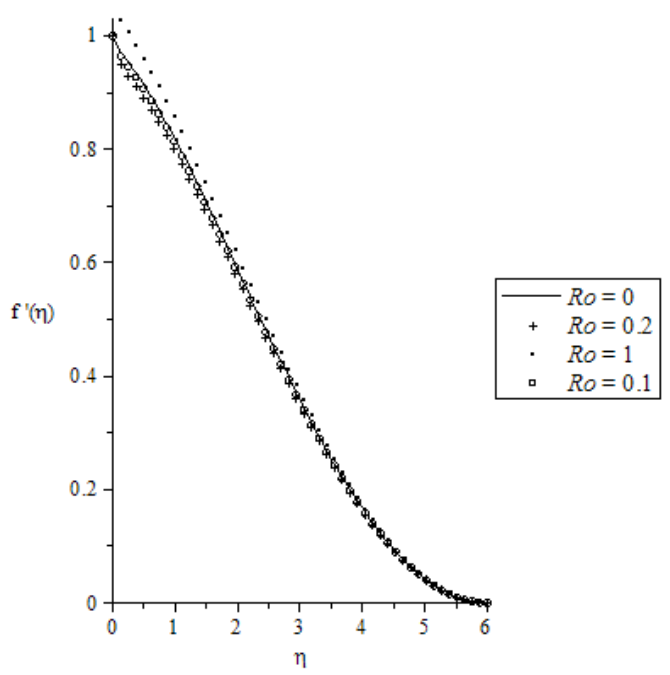

Figure 5. Velocity profiles for various values of $R o$ when $k_{1}=6.0, R_{0}=0.2, \theta_{r}=-1.0, \varepsilon=0.05$

\subsection{Temperature profiles}

The temperature profile $\theta(\eta)$ with $\eta$ for the flow field from the sheet for different values of controlling parameters are graphically shown in Figure 6 - 11. Figure 6 displays the influence of viscoelastic parameter $k_{1}$ on the temperature profile. It is observed that an increase in the viscoelastic parameter corresponds to an increase in the fluid temperature. However, the effect of increasing the magnetic interaction parameter $M n$ decreases the fluid temperature as shown in Figure 7. The thermal radiation $R_{0}$ and variable thermal conductivity $\varepsilon$ parameters have the same effects on the temperature profiles which is similar to their effects on the horizontal velocity profiles. Hence, increases in the values of these parameters enhance the fluid temperature as exhibited in Figures 8 and 9.

Observation from Figure 10 reveals the temperature profile $\theta(\eta)$ with $\eta$ from the sheet for different values of heat source/sink parameter $\beta$. It is observed that the temperature profile is reduced in the boundary layer for negative values of $\beta$ (heat sink) and is increased for positive values of $\beta$ (heat source). The physical interpretation of $\beta>0$ implies $T_{w}>T_{\infty}$, i.e. there will be a supply of heat to the flow region from the wall. Also, $\beta<0$ implies $T_{w}<T_{\infty}$, and there will be heat transfer from the flow to the wall. Hence, the influence of increasing the value of heat source/sink parameter $\beta$ corresponds to an increase of the temperature profile $\theta(\eta)$. Lastly, Figure 11 shows that an increase in the values of Prandtl number $\operatorname{Pr}$ decreases the temperature profile $\theta(\eta)$. An increase in the values of Prandtl number implies a decrease in thermal conductivity $k_{\infty}$. This is due to the fact that there would be a decrease of thermal boundary layer thickness with increasing values of Prandtl number.

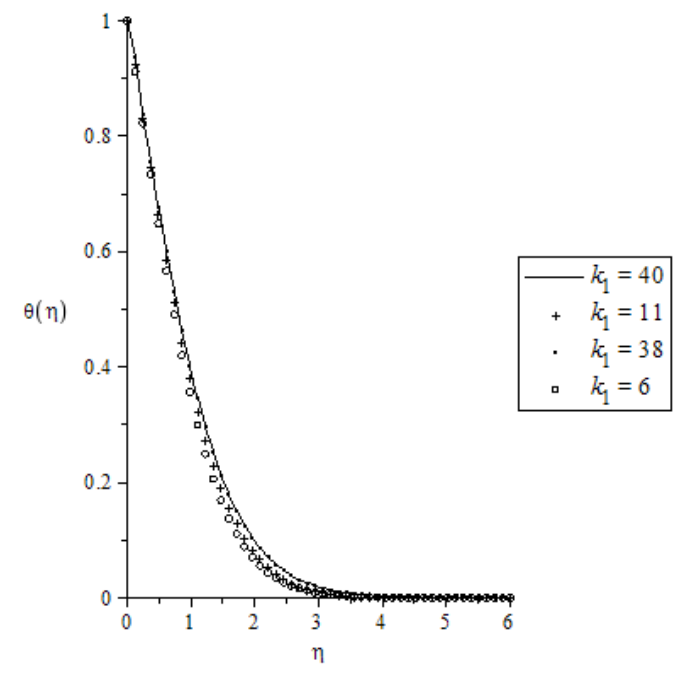

Figure 6. Temperature profiles for various values of $k_{1}$ when $\beta=0.2, R_{0}=0.2, \operatorname{Pr}=1, M n=0.5$

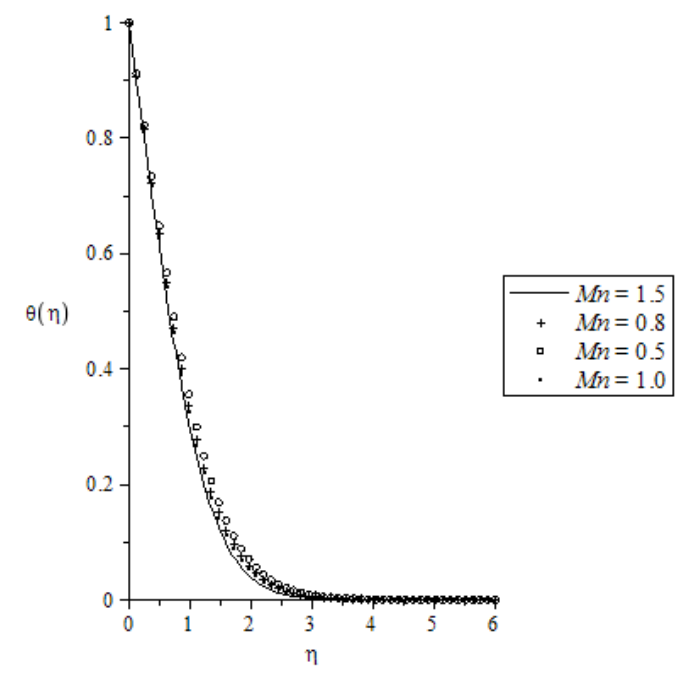

Figure 7. Temperature profiles for various values of $M n$ when $\beta=0.2, R_{0}=0.2, \operatorname{Pr}=1, k_{1}=6$ 


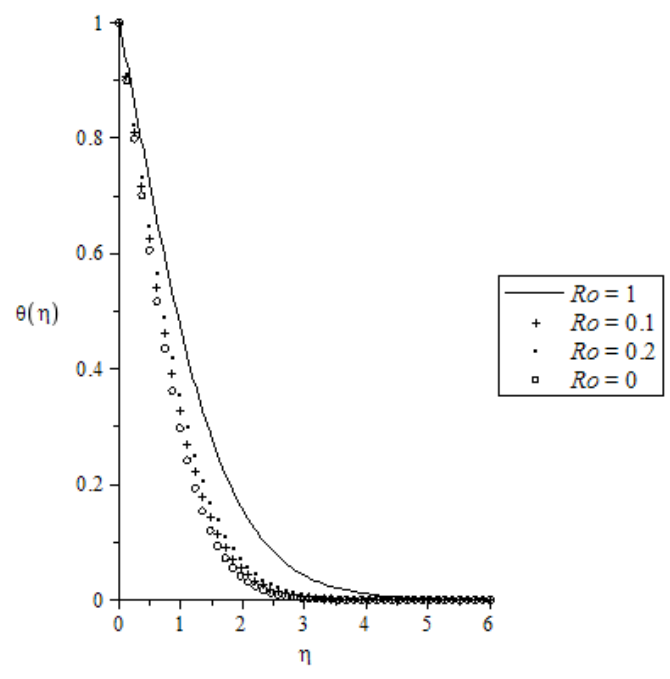

Figure 8. Temperature profiles for various values of $R o$ when $\beta=0.2, M n=0.5, \operatorname{Pr}=1, k_{1}=6$

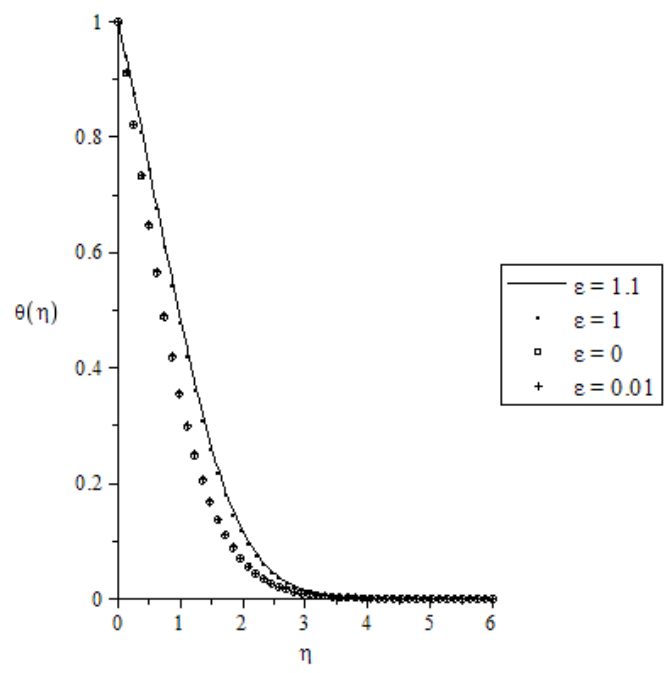

Figure 9. Temperature profiles for various values of $\varepsilon$ when $\beta=0.2, R_{0}=0.2, \operatorname{Pr}=1, k_{1}=6$

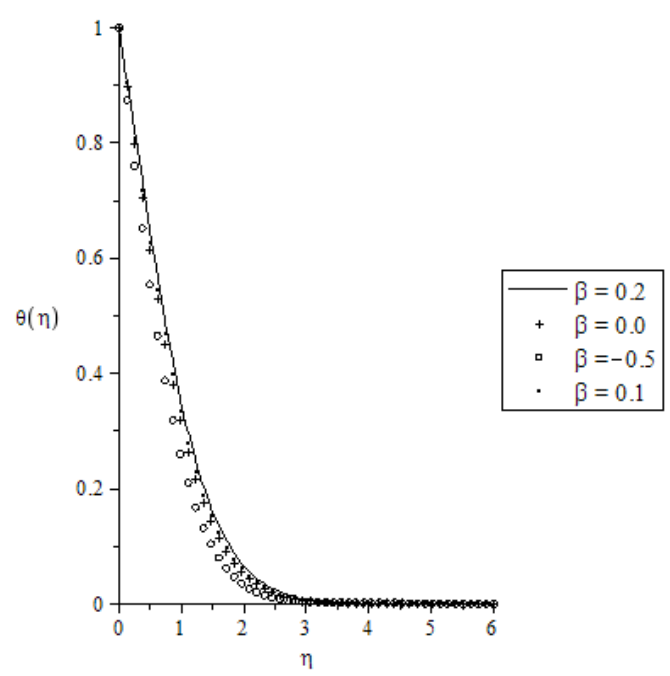

Figure 10. Temperature profiles for various values of $\beta$ when $M n=0.5, R_{0}=0.2, \operatorname{Pr}=1, k_{1}=6$

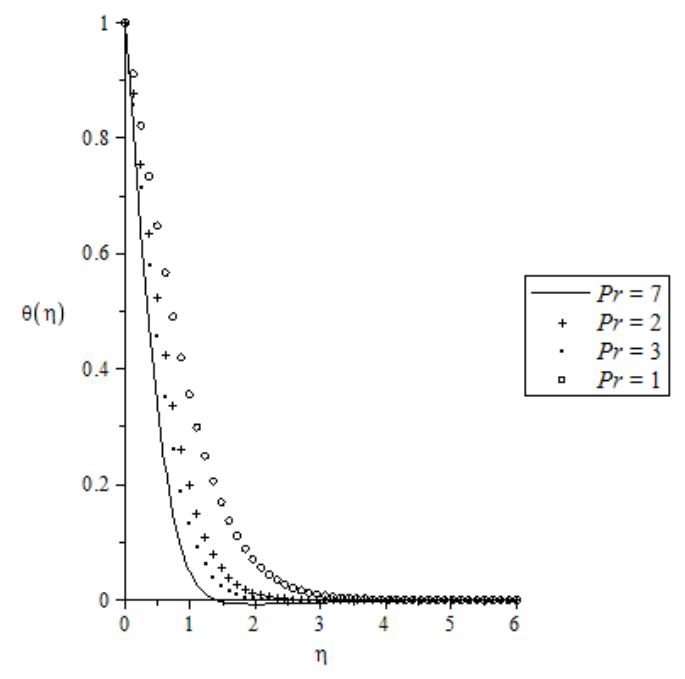

Figure 11. Temperature profiles for various values of $\operatorname{Pr}$ when $\beta=0.2, R_{0}=0.2, \varepsilon=0.005, k_{1}=6$

\subsection{Species concentration profiles}

The species concentration profile $\varphi(\eta)$ with $\eta$ for the flow field suffers a substantial change with the variation of flow parameters such as viscoelastic parameter $k_{1}$, magnetic interaction parameter $M n$, thermal radiation parameter $R_{0}$, variable thermal conductivity parameter $\varepsilon$, rate of chemical reaction $r$, Soret parameter $S r$, Schmidt number $S c$ and Prandtl number Pr. It is noticed from Figures $12-19$ that the values of the species concentration increase with an increase in viscoelastic parameter $k_{1}$, Soret parameter $\mathrm{Sr}$ and Prandtl number Pr. Thus, concentration boundary layer thickness increases with increasing values of these parameters. However, an increase in the values of magnetic interaction parameter $M n$, thermal radiation parameter $R_{0}$, variable thermal conductivity parameter $\varepsilon$, rate of chemical reaction parameter $r$ and Schmidt number $S c$ corresponds to a decrease in the species concentration. Therefore, this decreases the concentration boundary layer thickness as displayed by Figures $12-19$.

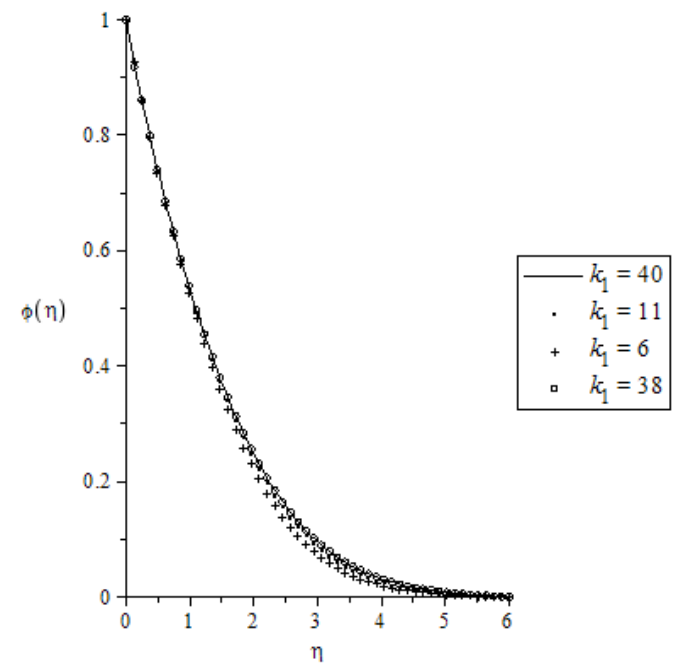

Figure 12. Concentration profiles for various values of $k_{1}$ when $S c=0.5, r=0.5, S r=0.5, M n=0.5$ 


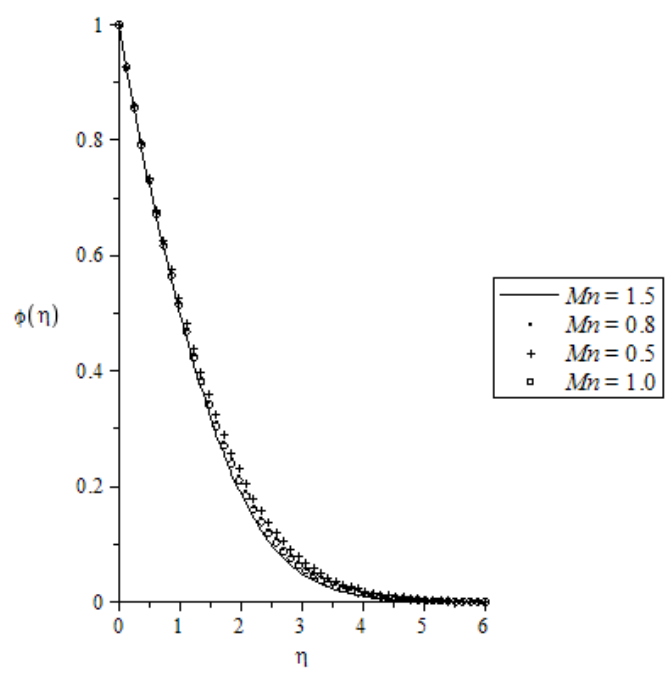

Figure 13. Concentration profiles for various values of $M n$ when $S c=0.5, r=0.5, S r=0.5, k_{1}=6.0$

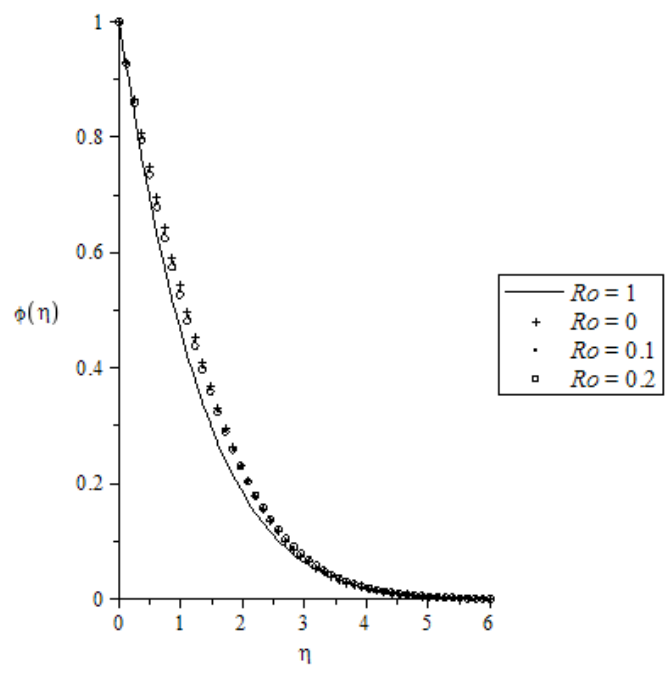

Figure 14. Concentration profiles for various values of $R o$ when $S c=0.5, r=0.5, S r=0.5, k_{1}=6.0$

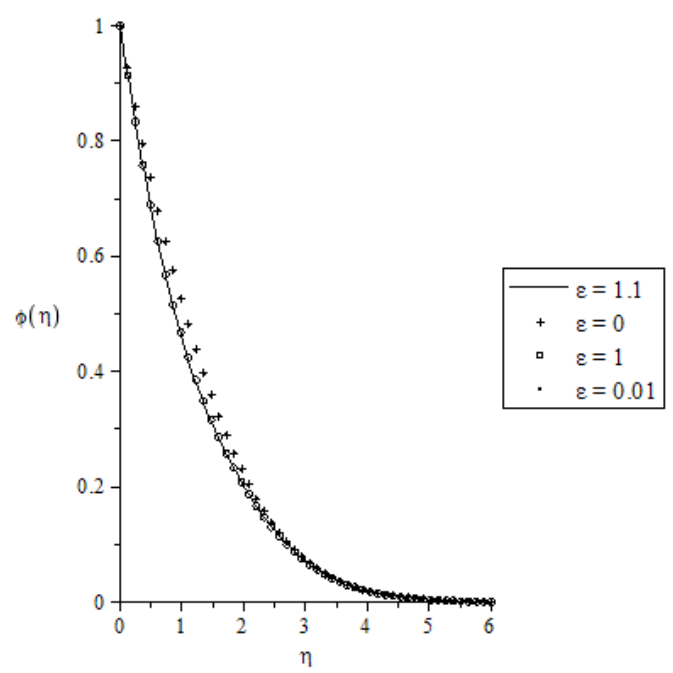

Figure 15. Concentration profiles for various values of $\varepsilon$ when $S c=0.5, r=0.5, S r=0.5, k_{1}=6.0$

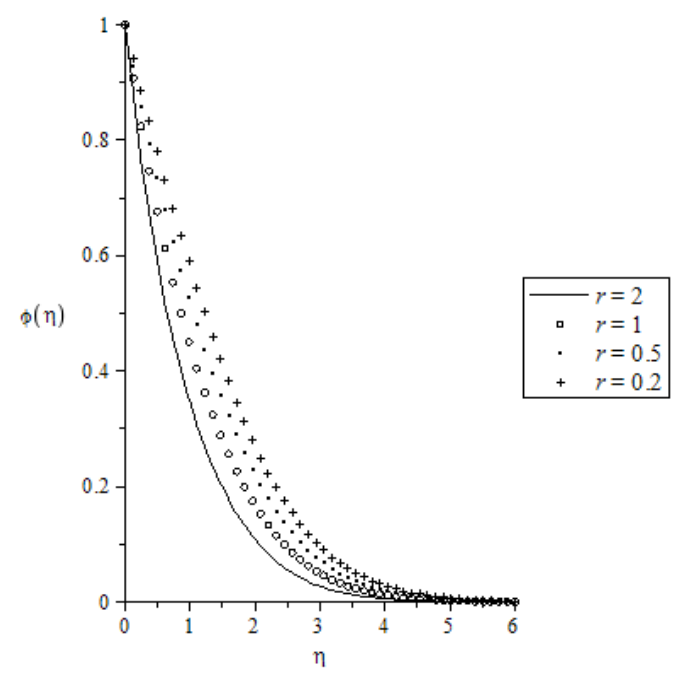

Figure 16. Concentration profiles for various values of $r$ when $S c=0.5, M n=0.5, S r=0.5, k_{1}=6.0$

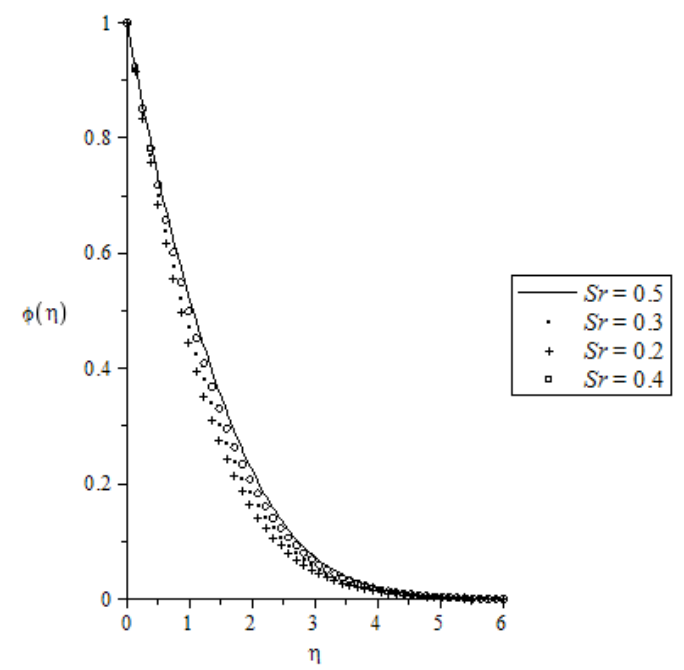

Figure 17. Concentration profiles for various values of $\mathrm{Sr}$ when $S c=0.5, r=0.5, M n=0.5, k_{1}=6.0$

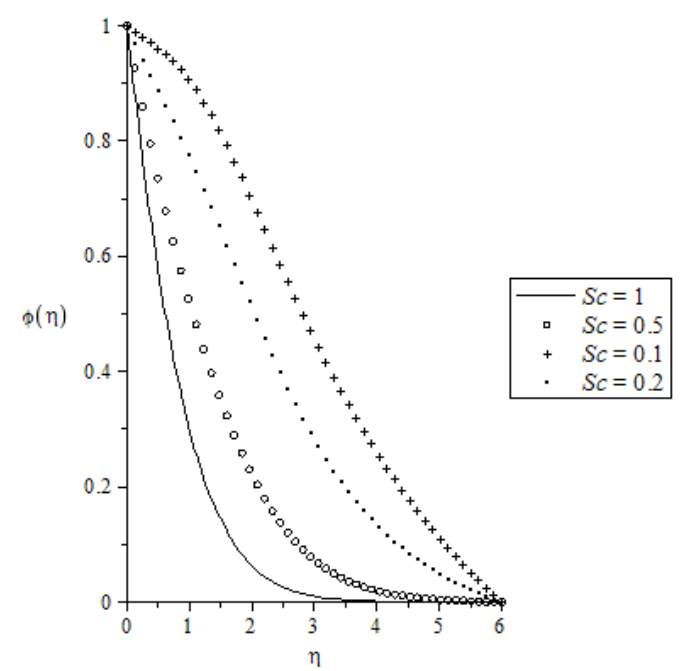

Figure 18. Concentration profiles for various values of $S c$ when $\mathrm{Mn}=0.5, r=0.5, \mathrm{Sr}=0.5, k_{1}=6.0$ 


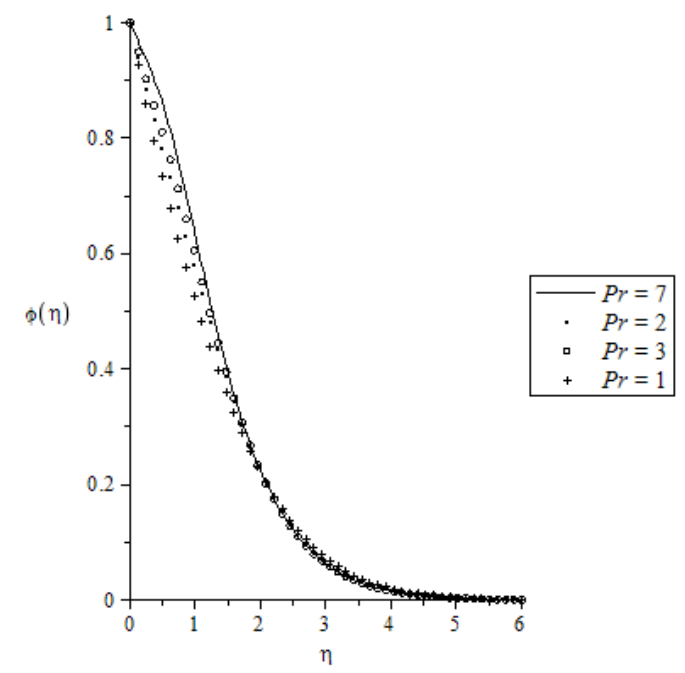

Figure 19. Concentration profiles for various values of $\operatorname{Pr}$ when $S c=0.5, r=0.5, S r=0.5, k_{1}=6.0$

Table 1. Numerical results (guess values obtained) for some values of sensitive parameters

\begin{tabular}{|c|c|c|c|c|c|c|c|c|c|c|c|c|}
\hline $\mathrm{Pr}$ & $\mathrm{R}$ & $\mathrm{Mn}$ & $\mathrm{Sc}$ & $\mathrm{r}$ & $\mathrm{Sr}$ & $\mathrm{B}$ & $\varepsilon$ & $\mathrm{K}_{1}$ & $\theta_{r}$ & $-f^{\prime \prime}(0)$ & $-\theta^{\prime}(0)$ & $-\varphi^{\prime}(0)$ \\
\hline \multirow[t]{3}{*}{1} & 0.5 & 0.1 & 0.2 & 0.2 & 0.1 & 0.2 & 0.05 & 3 & -1 & 0.14561814 & 0.81762062 & 0.40054048 \\
\hline & & & & & & & & & -5 & 0.15206379 & 0.81748185 & 0.40059741 \\
\hline & & & & & & & & & -10 & 0.15431441 & 0.81701603 & 0.40064599 \\
\hline \multirow[t]{3}{*}{1} & 0.5 & 0.1 & 0.2 & 0.2 & 0.1 & 0.2 & 0.05 & 3 & -1 & -0.14561814 & 0.81762062 & 0.40054048 \\
\hline & & & & & & & & 4 & & 0.01024139 & 0.78632225 & 0.38203173 \\
\hline & & & & & & & & 5 & & 0.08773145 & 0.76621793 & 0.37272378 \\
\hline \multirow[t]{3}{*}{1} & 0.5 & 0.1 & 0.2 & 0.2 & 0.1 & -0.5 & 0.05 & 3 & -1 & 0.13774017 & 1.00796373 & 0.38003817 \\
\hline & & & & & & 0.0 & & & & 0.14383081 & 0.87426499 & 0.39430502 \\
\hline & & & & & & 0.2 & & & & 0.14561814 & 0.81762062 & 0.40054048 \\
\hline \multirow[t]{3}{*}{1} & 0.5 & 0.1 & 0.2 & 0.2 & 0.1 & 0.2 & 0.0 & 3 & -1 & 0.14561529 & 0.84505740 & 0.39742565 \\
\hline & & & & & & & 0.05 & & & 0.14561814 & 0.81762062 & 0.40054048 \\
\hline & & & & & & & 0.2 & & & 0.14622407 & 0.74731520 & 0.40853662 \\
\hline \multirow[t]{3}{*}{1} & 0.0 & 0.1 & 0.2 & 0.2 & 0.1 & 0.2 & 0.05 & 3 & -1 & 0.13595004 & 0.16269642 & 0.45183547 \\
\hline & 0.5 & & & & & & & & & 0.14561814 & 0.81762062 & 0.40054048 \\
\hline & 0.8 & & & & & & & & & 0.14782272 & 1.03760785 & 0.37468119 \\
\hline 0.5 & 0.5 & 0.1 & 0.2 & 0.2 & 0.1 & 0.2 & 0.05 & 3 & -1 & 0.13776234 & 0.56684416 & 0.42723526 \\
\hline 1 & & & & & & & & & & 0.14561814 & 0.81762062 & 0.46054048 \\
\hline 1.5 & & & & & & & & & & 0.14761023 & 0.37860234 & 1.00455099 \\
\hline \multirow[t]{3}{*}{1} & 0.5 & 0.1 & 0.2 & 0.2 & 0.1 & 0.2 & 0.05 & 3 & -1 & 0.10610159 & 0.81083350 & 0.39613846 \\
\hline & & 0.2 & & & & & & & & 0.18453793 & 0.82434204 & 0.40498045 \\
\hline & & 0.5 & & & & & & & & 0.29518543 & 0.84581176 & 0.41794149 \\
\hline \multirow[t]{3}{*}{1} & 0.5 & 0.1 & 0.1 & 0.2 & 0.1 & 0.2 & 0.05 & 3 & -1 & 0.14561814 & 0.81762062 & 0.40054048 \\
\hline & & & 0.2 & & & & & & & 0.14561835 & 0.81763016 & 0.34390965 \\
\hline & & & 0.4 & & & & & & & 0.14561863 & 0.81764322 & 0.23064138 \\
\hline \multirow[t]{3}{*}{1} & 0.5 & 0.1 & 0.2 & 0.2 & 0.1 & 0.2 & 0.05 & 3 & -1 & 0.14561814 & 0.81762062 & 0.40054048 \\
\hline & & & & 1 & & & & & & 0.14561781 & 0.81759919 & 0.55925017 \\
\hline & & & & 2 & & & & & & 0.14561731 & 0.81757081 & 0.71307436 \\
\hline \multirow[t]{3}{*}{1} & 0.5 & 0.1 & 0.2 & 0.2 & 0.5 & 0.2 & 0.05 & 3 & -1 & 0.14561639 & 0.81755237 & 0.71355693 \\
\hline & & & & & 1.0 & & & & & 0.14561105 & 0.81740894 & 1.07606348 \\
\hline & & & & & 2.0 & & & & & 0.14558588 & 0.81706787 & 1.60028221 \\
\hline
\end{tabular}

\section{CONCLUSION}

The present study focuses on the effect of thermal radiation, chemical reaction, variable viscosity and an internal uniform magnetic field on the flow, temperature and species concentration distribution of a magnetohydrodynamic nonNewtonian viscoelastic fluid over a stretching sheet with variable thermal conductivity in the presence of the nonuniform heat source/sink and thermal diffusion. Using appropriate similarity transformations, the formulated governing equations are transformed and then solved 
numerically by employing Runge-Kutte-Fehlberg scheme with shooting technique. The numerical results show the influences of thermal diffusion and radiation, variable viscosity and other physical controlling parameters on the flow, heat and mass characteristics. Therefore, results of physical interest on velocity, temperature and species concentration distributions of flow field as well as local shear stress, wall temperature and species concentration gradients are summarized below:

i. The influence of viscoelastic parameter is to decrease velocity distribution and increase both temperature and species concentration distribution in the boundary layer. Contrast effect is seen for magnetic field interaction parameter on the velocity, temperature and species concenntration profiles. It is also observed that the velocity distribution decreases with increasing values of fluid viscosity parameter;

ii. As the intensity of the thermal radiation and variable thermal conductivity parameters increase, the velocity and temperature profiles increase. However, an increase in thermal radiation and variable thermal conductivity parameter is found to decrease with the species concentration profile;

iii. The effect of Prandtl number is found to increase and decrease the thermal and species concentration boundary layer thicknesses respectively. Also, as the value of Schmidt number increases, it has the influence of decreasing the species concentration boundary layer thickness;

iv. The temperature distribution is lower throughout the boundary layer for heat-sink parameter and higher for heat-source parameter. The chemical reaction and thermal diffusion parameters have opposite effects on the species concentration profiles;

v. The effect of Prandtl number, variable thermal conductivity, heat source/sink and magnetic interaction parameters is to increase skin-friction coefficient and wall concentration gradient while the viscoelastic parameter decreases the wall temperature and concentration gradients;

vi. The wall temperature gradient increases with increase in the values of Schmidt number, thermal radiation and magnetic interaction parameters but the reverse is the case with rate of chemical reaction, variable thermal conductivity, heat source/sink and thermal diffusion parameters.

\section{ACKNOWLEDGMENT}

The authors acknowledge the thoughtful comments of the referees.

\section{REFERENCES}

[1] Abel, M. S. Siddheshwar, P. G. and Nandeppanavar Mahentesh, M., "Heat transfer in a viscoelastic boundary layer flow over a stretching sheet with viscous dissipation and non-uniform heat source," Int. J. Heat Mass Transfer, vol. 50, pp. 960-966, 2007. DOI: $10.1016 /$ j.ijheatmasstransfer.2006.08.010.

[2] Abel, M. S. and Nandeppanavar M. M., "Heat transfer in MHD viscoelastic boundary layer flow over a stretching sheet with non-uniform heat source/sink,"
Commun. Nonlinear Sci. Numer Simulat., vol. 14, pp. 2120-2131, 2009. DOI: 10.1016/j.cnsns.2008.06.004.

[3] Abel, M. S. and Nandeppanavar, M. M, "Heat transfer in MHD viscoelastic boundary layer flow over a stretching sheet with space and temperature-dependent heat source," Int. J. Appl. Mech. Eng., vol. 13, no. 2, pp. 293-309, 2008.

[4] Hayat, T., Javed, T. and Abbas, Z., "Slip flow and heat transfer of a second grade fluid past a stretching sheet through a porous space," Int. J. Heat Mass transfer, vol. 51, pp. 4528-4534, 2008. DOI: 10.1016/j.ijheatmasstransfer.2007.12.022.

[5] Khan, S. K., Abel, M. S., Sonth, R. M., "Viscoelastic MHD flow, heat and mass transfer over a porous stretching sheet with dissipation of energy and stress work," Heat Mass Transfer , vol. 40, pp. 47-57, 2003.

[6] Kesavaiah, D. C., Satyanarayana, P. V. and Venkaturamana, S., "Effects of the chemical reaction and radiation absorption on an unsteady MHD convective heat and mass transfer flow past a semiinfinite vertical permeable plate embedded in a porous medium with heat source and suction," Int. J. of Appl. Math and Eng., vol. 7, no. 1, pp. 52-69, 2011.

[7] Chang, T. B., Mahmood, A., Beg, O. A., Narahari, M., Islam, M. N. and Ameen, F., "Numerical study of transient free convective mass transfer in a Walter's B viscoelastic flow with wall suction," Commun Nonlinear Sci. and Numer. Simulat., vol. 16, pp. 216-225, 2011.

[8] Vajravelu, K. and Rollins, D., "Hydromagnetic flow of a second grade fluid over a stretching sheet," Appl. Math. Comp., vol. 148, pp. 783-791, 2004. DOI: 10.1016/S0096-3003(02)00942-6.

[9] Datti, P. S., Prasad, K. V., Abel, M. S. and Joshi, A., "MHD viscoelastic fluid flow over a non-isothermal stretching sheet," Int. J. Eng. Sci., vol. 42, pp. 935946, 2004.

[10] Cortell, B. F., "Viscoelastic fluid flow and heat transfer over a stretching sheet under the effects of non-uniform heat source, viscous dissipation and thermal radiation," Int. J. Heat Mass transfer, vol. 50, pp. 3152-3162, 2007.

[11] Abel, M. S., Siddheshwar, P. G. and Mahantesh, M. Nandeppanavar. "Heat transfer in a viscoelastic boundary layer flow over a stretching sheet with viscous dissipation and non-uniform heat source," Int. J. Heat Mass Transfer, vol. 50, pp. 960-966, 2007. DOI: $10.1016 /$ j.ijheatmasstransfer.2006.08.010.

[12] Sajid, M., Ahmad, I., Hayat, T.and Ayub, M., "Unsteady flow and heat transfer of a second grade fluid over a stretching sheet," Commun. Non-linear Sci. Numer. Simulat., vol. 14, pp. 96-10, 2009. DOI: 10.1016/j.cnsns.2007.07.014.

[13] Hayat, T., Saif, S. and Abbas, Z., "The influence of heat transfer in an MHD second grade fluid film over an unsteady stretching sheet," Phys. Lett., vol. A, no. 372, pp. 2400-2408, 2008. DOI: 10.1016/j.physleta.2008.03.066.

[14] Massoudi, M. and Maneschy, C. E., "Numerical solution to the flow of a second grade fluid over a stretching sheet using the method of quasilinearization," Appl. Math. Comput., vol. 149, pp. 165173, 2004. DOI: 10.1016/S0096-3003(02)00963-3.

[15] Sudheer Babu M. and Satya Narayana, P. V., "Effects of Chemical reaction and radiation absorption on free 
convection flow through porous medium with variable suction in the presence of uniform magnetic field," Journal of Heat and Mass Transfer, vol. 3, pp. 219234, 2009.

[16] Gireesh K. J., Satyanarayana, P. V. and Ramakrishna, S., "Effects of Chemical reaction and mass transfer on MHD unsteady free convection flow past an infinite vertical plate with constant suction and heat sink," Ultra Science, vol. 21, no. 3, pp. 639-650, 2009.

[17] Kulandaivel, T., Loganathan, P. and Muthucumaraswamy, R. "Chemical reaction on moving plate with constant mass flux in the presence of thermal radiation," Int'l Journal of Applied Mathematics and Mechanics, vol. 5, pp. 84-95, 2009.

[18] Gireesh K. J. and Satyanarayana, P. V., "Mass transfer effects on MHD unsteady free convective Walter's memory flow with constant suction and heat sink," Int. J. Appl. Maths. and Mech., vol. 9, no. 19, pp. 97-109, 2011.

[19] Hayat, T. and Qasim, M. "Radiation and Magnetic field effects on the unsteady mixed convection flow of a second grade fluid over a vertical stretching sheet". Int. J. Numer. Meth. Fluids, vol. 66, pp. 820-832, 2011. DOI: 10.1002/fld.2285.

[20] Bhargava, R., Sharma, S., Takhar, H. S., Beg, O. A. and Bhargava, P., "Numical solutions for micropolar transport phenomena over non-linear stretching sheet". Nonlinear Analy. Model. Cont., vol. 12, pp. 45-63. 2007.

[21] Singh, A. K. "Heat source and radiation effects on magneto-convection flow of a viscoelastic fluid past a stretching sheet: analysis with Kummer's function". Int. Commun. Heat and Mass Transfer, vol. 35, pp. 637-642, 2008.

DOI: 10.1016/j.icheatmasstransfer.2008.01.009.

[22] Aman, F. and Ishak, A., "Hydromagnetic flow and heat transfer adjacent to a stretching vertical sheet with prescribed surface heat flux," Heat Mass Transfer, vol. 46, pp. 615-620, 2010. DOI: 10.1007/s00231-0100606-6.

[23] Abbas, Z. and Hayat, T., "Stagnation slip flow and heat transfer over a non-linear stretching sheet," Numer. Meth. Part. Differ. Equat., vol. 27, pp. 302314, 2011.

[24] Pal, D. and Mondal, H., "Soret and Dufuour effects on MHD non-Darcian mixed convection heat and mass transfer over a stretching sheet with non-uniform heat source/sink," Physica B., vol. 85, pp. 941-951, 2010.

[25] Malashestty, M. S. and Umavathi, J. C., "Two-phase magnetohydrodynamic flow and heat transfer in an inclined channel," Int. J. Multiphase flow, vol. 23, pp. 545-560, 1997.

[26] Malashestty, M. S., Umavathi, J. C. and Prathap, Kumar J., "Magnetoconvection of two-immiscible fluids in vertical enclosure," Heat \& Mass Transfer, vol. 12, pp. 977-993, 2011.

[27] Cortell, R., "Flow and heat transfer of an electrically conducting fluid of second grade over a stretching sheet subject to suction and a transverse magnetic field," Int. J. Heat \& Mass Transfer, vol. 49, pp. 18511856 , 2006.

DOI: 10.1016/j.ijheatmasstransfer.2005.11.013.

[28] Seddeek, M. A., "Heat and mass transfer on a stretching sheet with magnetic field in a viscoelastic fluid flow through a porous medium with heat source or sink," Comput. Mater. Sci., vol. 38, pp. 781-787, 2007.

[29] Emad, M. A. and Mohammed A. E., "Blowing or suction effect on hydromagnetic heat transfer by mixed convection from an inclined continuously stretching surface with internal heat generation/absorption," Int. J. Thermal Sci., vol. 43, pp. 709-719, 2004.

[30] Grosan, T. and Pop, I., "Thermal radiation effect on fully developed mixed convection flow in a vertical channel," Tech. Mech., vol. 1, pp. 37-47, 2007.

[31] Srinivas, S. and Muthuraj, R., "Effects of thermal radiation and space porosity on MHD mixed onvection flow in a vertical channel using homotopy analysis method," Commun. Nonlinear Sci. Numer. Simulat., vol. 51, pp. 2098-2108, 2010.

[32] Joshi, N. and Kumar, M., "The combined effects of chemical reaction, radiation, MHD on mixed convection heat and mass transfer along a moving surface," Applications and Applied Maths., vol. 5, no.10, pp.1631-1640, 2010.

[33] Eldabe, N. T., El-Saka, A. G. and Fouad, A., "Thermal diffusion and diffusion thermo effects on mixed freeforced convection and mass transfer boundary layer flow for non-Newtonian fluid with temperature dependent viscosity," Appl. Math. And Comput., vol. 152, pp. 867-883, 2004. DOI: 10.1016/S00963003(03)00603-9.

[34] Anghel, M., Takhar, H. S. and Pop, I, "Dufuour and Soret effects on free-convection boundary layer over a vertical surface embedded in a porous medium," Studia Universitatis Babes-Bolya, Mathematica, vol. XLV, pp.11-21, 2000.

[35] Beg, A. O., Bakier, A.Y. and Prasad, "Numerical study of free convection magnetohydrodynamic heat and mass transfer from a stretching surface to a saturated porous medium with Soret and Dufour effects," Computational Material Sci., vol. 46, pp. 57-65, 2002.

[36] Herwing, H. and Gersten, K., "The effect of variable properties on laminar boundary layer flow," Warme Stoffubertrag, vol. 20, pp. 47-57, 1986.

[37] Lai, F. C. and Kulacki, F. A. "The effect of variable viscosity on convective heat transfer along a vertical surface in a saturated porous medium," Int. J. Heat Mass Transfer, vol. 33, pp. 1028-1031, 1990.

[38] Pop, I., Gorla, R.S.R. and Rashidi, M., "The effect of variable viscosity on flow and heat transfer to a continuous moving flat plate," Int. J. Eng. Sci., vol. 30, pp. 1028-1031, 1992. DOI: 10.1016/00207225(92)90115-W.

[39] Abel, M. S., Khan, S. K. and Prasad, K. V., "Study of viscoelastic fluid and heat transfer over a stretching sheet with variable viscosity," Int. J. Non-Linear Mech., vol., 37, pp. 81-88, 2002.

[40] Mukhopadhyay, S. and Layek, G. C., "Effect of thermal radiation and variable fluid viscosity on free convective and heat transfer past a porous stretching surface," Int. J. Heat Mass Transfer, pp. 2167-2178, 2008. DOI: $10.1016 /$ j.ijheatmasstransfer.2007.11.038.

[41] Pantokratoras, A., "Further results on the variable viscosity on the flow and heat transfer to a continuous moving flat plate," Int. J. Eng. Sci., vol. 42, pp. 18911896, 2004. DOI: $10.1016 / j . i j e n g s c i .2004 .07 .005$.

[42] Mukhopadhyay, S. and Layek, G. C. "Effect of thermal radiation and variable fluid viscosity on free convective and heat transfer past a porous stretching 
surface," Int. J. Heat Mass Transfer, pp. 2168-2178, 2008. DOI: $10.1016 /$ j.ijheatmasstransfer.2007.11.038.

[43] Siddappa, B. and Abel, S. M. "Non-Newtonian flow past a stretching surface," Z. Angew Math. Phys., vol. 36, pp. 890-892, 1985.

[44] Rivlin, R. S. and Ericksen. J. L., "Stress Deformation Relation for Isotropic Materials," J. Rat. Mech. Anas, vol. 4, pp. 323-425, 1955.

[45] Dandapat, B. S., Holmedal, L. E. and Anderson, H. L., "Stability of flow of a viscoelastic fluid over a stretching sheet," Arch Mech., vol. 46, no.6, pp. 829838, 1994.

[46] T. Y. Na, Computational Methods in Engineering: Boundary Value Problems, New York: Academic Press, pp. 19-28, 1979.

[47] Chaim, T. C., "Heat transfer with variable thermal conductivity in a stagnation-point flow towards a stretching sheet," Int. Commun. Heat Mass Transfer, vol. 23, pp. 239-248, 1996.

[48] Salem, A. M., "Variable viscosity and thermal conductivity effects on MHD flow and heat transfer in viscoelastic fluid over a stretching sheet," Phys Lett., vol. A, pp. 315-322, 2007. DOI: 10.1016/j.physleta.2007.04.104.

[49] B. M. Quinn, Thermal Radiative Heat Transfer Properties, New York, Wiley,pp. 453-463, 1992.

[50] Rajagopal, K. R., Na, T. Y. and Gupta, A. S., "Flow of visco-elastic fluid due to stretching sheet," Rheol. Acta, vol. 23, pp. 213-215, 1984.

[51] Rajagopal, K. R., Na, T. Y. and Gupta, A. S., "A nonsimilar boundary layer on stretching sheet in a non-

Newtonian fluid with uniform free stream," Math. Phys. Sci., vol. 21, no.2, pp. 189-200, 1987.

[52] Mahapatra, T. R. and Gupta, A. S., "Stagnation-point flow of visco-elastic fluid towards a stretching surface," Int. J. Non-Linear Mechanics, vol. 39, pp. 811-820, 2004.

\section{NOMENCLATURE}

$\mathrm{u}$ and $\mathrm{v}$

$\mathrm{k}_{\mathrm{o}}$

$\mathrm{D}_{\mathrm{m}}$

$\mathrm{C}_{\mathrm{P}}$

$\mathrm{k}$

$\mathrm{K}_{\mathrm{T}}$

$\operatorname{Pr}$

$\mathrm{Sc}$

Ro

$\mathrm{Sr}$

\section{Greek symbols}

Bo

$\sigma$

$\Theta_{\mathrm{r}}$

$\mu$

$\rho_{\infty}$
Fluid velocity components in the $\mathrm{x}$ and $\mathrm{y}$ directions respectively.

Coefficient of viscoelasticity

Coefficient of mass diffusivity

specific heat, J. kg-1. K-1

thermal conductivity, W.m-1. K-1

thermal diffusion parameter, $\mathrm{m} 2$. s-1

Prandtl number

Schmidtl number

Thermal radiation parameter

Thermal diffusion parameter
Magnetic field strength

Electrical conductivity

Fluid viscosity parameter

Coefficient of viscosity, kg. m-1.s-1

Constant fluid density 